\title{
Clinical Study \\ Effects of a Teacher-Centred, School-Based Intervention Program on Health Behavior and Cardiovascular Disease Risk in Elementary School Children
}

\author{
Clemens Drenowatz, ${ }^{1}$ Olivia Wartha, ${ }^{1}$ Susanne Brandstetter, ${ }^{1,2}$ and Jürgen M. Steinacker ${ }^{1}$ \\ ${ }^{1}$ Division of Sport and Rehabilitation Medicine, Ulm University Medical Center, Frauensteige 6, 89075 Ulm, Germany \\ ${ }^{2}$ Institute for Epidemiology and Preventive Medicine, University of Regensburg, 93053 Regensburg, Germany \\ Correspondence should be addressed to Clemens Drenowatz; clemens.drenowatz@uni-ulm.de
}

Received 18 June 2013; Accepted 2 August 2013

Academic Editors: W. B. Hansen, T. Morken, P. J. Naylor, and S. M. Pezzotto

Copyright (C) 2013 Clemens Drenowatz et al. This is an open access article distributed under the Creative Commons Attribution License, which permits unrestricted use, distribution, and reproduction in any medium, provided the original work is properly cited.

\begin{abstract}
The increasing prevalence of overweight and obese children along with accompanying comorbidities has prompted an early acknowledgement of a healthy lifestyle. The purpose of this study was to examine the effect of a teacher-centered, school-based intervention on cardiovascular disease (CVD) risk and health behavior in elementary school children. 935 first- and second-grade children in southwest Germany provided valid data at baseline and follow-up. Trained technicians measured height and weight along with blood pressure, cholesterol, and intra-abdominal fat to determine CVD risk. Parent questionnaires were used to assess children's health behavior. Within one year CVD risk declined in the intervention group, particularly due to an attenuation of the age-related increase in mean arterial pressure. The age-related decline in habitual sports participation was attenuated, and children in the intervention groups displayed higher odds of playing outside. Further, the consumption of sugar-sweetened beverages declined in the intervention group, and TV time remained stable, while it increased in the control group. These results indicate that a teacher-centered intervention positively affects health behavior and CVD risk. The incorporation of the intervention by the classroom-teacher should allow for a sustainable participation, which may result in more pronounced effects over time.
\end{abstract}

\section{Introduction}

The prevalence of overweight and obesity has increased in many industrialized countries [1], and obesity has already been declared as one of the leading threats for public health [2]. Of particular concern is the increasing number of overweight or obese children and adolescents [1], which has lead to the occurrence of various cardiovascular and metabolic disease risks that were previously only observed in adults [3]. Further, overweight children are more likely to become overweight adults [4] and, therefore, have an increased risk for all-cause mortality [5]. In addition to the impact on individual health and quality of life, the increased risk for various chronic diseases associated with excess body fat puts a significant economic burden on the society [6]. While physiologic and genetic aspects need to be considered, high energy intake and insufficient physical activity (PA) have been primarily emphasized as part of the problem [7]. As lifestyle patterns are established at a young age and health behavior has been shown to track into adulthood [8], primary prevention programs that promote sufficient PA and healthy eating patterns at an early age are warranted. Children and adolescents have also been shown to be more responsive to health promotion programs [9], which would result in a higher success rate of specific interventions compared to adults who have difficulties in adopting and adhering to a healthy lifestyle [10].

School-based intervention programs have become popular as children spend a considerable amount of time in schools, and a large number of children from various cultural and socioeconomic backgrounds can be reached [6]. Most intervention efforts have focused on rising awareness or changing attitudes and motivation towards physical activity, sedentary behaviour, and diet [11]. Despite considerable 
efforts, the success of school-based interventions concerning health behavior or body composition, however, has been limited [12, 13]. Kropski et al. argue that short intervention periods, as well as methodological issues including a lack of randomized controlled study designs, contribute to a lack of conclusive evidence regarding the effectiveness of school-based interventions on weight-related outcomes [14]. The focus on body weight or body composition as primary outcome, rather than on behavioral changes or other CVD risk factors, may also be problematic. Due to growth-related changes in body composition, especially around puberty, changes in body composition may not necessarily reflect intervention effects adequately, and in a meta-analysis, Harris et al. did not show any difference in BMI between control and physical activity intervention groups [15]. Even though body composition affects CVD risk [16], other risk factors, such as blood pressure, cholesterol levels, or intra-abdominal fat, need to be considered [17]. The focus of this study, therefore, was to examine the intervention effects of a teacherimplemented intervention program on health behavior and CVD risk, rather than body composition.

\section{Methods}

2.1. Subjects. Thirty-two schools with 64 first- and secondgrade classes in southwest Germany agreed to participate in URMEL-ICE (Ulm Research on Metabolism, Exercise and Lifestyle in Children). The study protocol was approved by the Ministry of Culture and Education of the state of Baden-Württemberg, Stuttgart as well as the institutional ethics committee and is in accordance with the declaration of Helsinki. After ensuring participation, schools were randomly assigned to either the intervention or the one-year waiting-control group. Parental consent as well as child assent was obtained for 1119 (78\%) elementary school children. The average age at baseline was $7.6 \pm 0.5$ years. 935 (445 intervention, 490 control) children provided valid data at both measurement times, baseline and one-year follow-up. Due to missing data on various measurements, sample size, however, varies for individual analyses.

2.2. Intervention. The intervention mapping protocol (IMP) [18] was used to guide the program development. A major aspect of the program was the implementation of the intervention by regular classroom teachers, who were familiarized with the program via 4 seminars prior to the beginning of the intervention. The intervention did not require additional time for physical education and was developed in cooperation with experienced teachers to be in accordance with the current curriculum to ensure the applicability of the materials in a normal classroom setting. Materials consisted of a total of 29 teaching units per year (30-60 minutes each) and various short activities, which could be implemented in the classroom. Teachers were asked to provide 2 in-class activity blocks per day, lasting 5-7 minutes each. Further, materials for parental information and 6 family homework assignments were included to facilitate parental involvement. The homework assignments informed parents about the current health-related content taught in class and promoted an active engagement of parents in their children's leisure activities. Teachers were also provided with materials for 2 parent-teacher meetings. The emphasis of the intervention was on behavior change, specifically focusing on increasing PA, reducing screentime, and decreasing the consumption of sugar-sweetened beverages. A special emphasis was put on the promotion of healthy alternative behaviors such as active transportation and active play.

2.3. Anthropometrics. Measurements were taken during the fall of the school year prior to the intervention and again one year later. All measurements were performed in a similar fashion at baseline and follow-up. Anthropometric measurements were taken by trained staff from the outpatient clinic of Ulm Children's Hospital according to standard procedures with the children wearing only underwear. Using a stadiometer (Ulm Stadiometer, Busse Design, Ulm, Germany), height $(\mathrm{cm})$ was measured to the nearest $0.1 \mathrm{~cm}$ and weight $(\mathrm{kg})$ was measured to the nearest $0.1 \mathrm{~kg}$ using a balance beam scale (Seca, Hamburg, Germany). BMI was calculated $\left(\mathrm{kg} / \mathrm{m}^{2}\right)$ and converted to BMI percentiles (BMIPCT) using German reference values [19]. Overweight and obesity were subsequently determined at a BMIPCT above the 90th and 97th percentiles, respectively [19].

2.4. CVD Risk. CVD risk was determined based on mean arterial pressure (MAP), the ratio of total cholesterol to HDL (TC:HDL), and intra-abdominal fat (IAF). Blood pressure was measured twice with the child in a seated position according to the guidelines of the National High Blood Pressure Education Program [20]. MAP was calculated $(\mathrm{MAP}=$ diast. $\mathrm{BP}+1 / 3 *$ syst. $\mathrm{BP})$ using the mean of the two measurements. Blood samples obtained via venous puncture were processed shortly after withdrawal. Following standard procedures, chemical analyses were performed to determine total cholesterol, HDL-cholesterol, and LDLcholesterol. IAF was assessed via sonographic measures of intra-abdominal depth using a $3.5 \mathrm{MHz}$ convex transducer. Two to three measurements were performed by a single well trained technician with the child lying in a supine position following recommendations by Armellini et al. [21]. Specifically, measurements were taken along the linea alba at the height of the branching of the arteria mesenterica at the end of the expiration. An overall CVD risk score was calculated using principal component factor analysis including MAP, TC:HDL, and IAF, which resulted in a single factor (Eigenvalue $=1.22$ ) that explained $40.6 \%$ of the variance at baseline. Component loadings were $0.76,0.65$, and 0.46 for IAF, TC : HDL, and MAP, respectively. At follow-up, the Eigenvalue of the CVD risk score was 1.26 and explained $42.0 \%$ of the variance. Component loadings for the follow-up CVD risk score were $0.71,0.62$, and 0.61 for IAF, TC: HDL, and MAP, respectively.

2.5. Health Behavior. Parental questionnaires were used to assess children's physical activity, sports participation, sedentary behaviour, breakfast habits, and the consumption of 
sugar-sweetened beverages. Questions were based on the KiGGS survey, which assessed health behavior in a representative sample of 18,000 German children and adolescents. The KiGGS questionnaire was developed based on expert evaluation of existing national and international studies and data sources [22]. Specifically, parents were asked about their children's participation in sports, time spent playing outside, and walking and active transport. Further, parents reported whether their child had breakfast prior to going to school, the consumption of sugar-sweetened beverages, and time spent watching TV and playing computer.

2.6. Statistical Analysis. Descriptive statistics were calculated and repeated measures ANCOVA, controlling for age and sex, were performed to assess time by intervention effects on CVD risk. To control for the effect of body composition on CVD risk, BMIPCT was added as a covariate in a second analysis. Further, differences in CVD risk at follow-up were analysed via ANCOVA, controlling for sex, age, and baseline values. In addition, dependent $t$-tests were used to examine changes over time in CVD risk and health behaviour in each group separately. For the categorical variables regarding health behaviour, odds ratios at follow-up, adjusted for sex, age, and baseline values, were calculated as well. Statistical analyses were performed using SPSS 19.0 with a significance level set at $P<0.05$ using Bonferroni adjustment for multiple analyses.

\section{Results}

There were no differences in age, BMIPCT, or CVD risk factors at baseline between children included in the analysis and those who did not provide data at follow-up. Table 1 shows the descriptive characteristics of the sample with data during baseline and follow-up, separately for the control and intervention groups. No sex differences were shown for BMIPCT and CVD risk score at baseline and follow-up. Boys, however, had higher IAF, while girls had a higher TC : HDL ratio at both measurements. At baseline boys also displayed significantly higher MAP compared to girls.

Repeated measures ANCOVA, controlling for sex and age, did not show any significant results concerning BMIPCT. For the overall CVD risk score, a significant time by intervention effect occurred (Wilks $\lambda=0.99, F(1,639)=8.72$, $P<0.01$, partial $\left.\varepsilon^{2}=0.01\right)$ without significant main effects for either time or intervention. Concerning individual CVD risk factors, a time by intervention effect was shown for MAP (Wilks $\lambda=0.98, F(1,898)=16.91, P<0.01$, partial $\varepsilon^{2}=0.02$ ) and IAF (Wilks $\lambda=1.00, F(1,898)=4.24, P=0.04$, partial $\left.\varepsilon^{2}=0.01\right)$. There were no main effects for the intervention in any of the CVD risk factors, but there was a significant main effect for time for IAF (Wilks $\lambda=0.99, F(1,898)=$ 7.59, $P<0.01$, partial $\left.\varepsilon^{2}=0.01\right)$. Results were similar for MAP and TC:HDL ratio after additionally controlling for BMIPCT. For IAF, however, the time by intervention effect was no longer present, but main effects were observed for time (Wilks $\lambda=1.00, F(1,897)=4.46, P=0.04$, partial $\left.\varepsilon^{2}=0.01\right)$ as well as intervention $(F(1,897)=8.56, P<0.01$,
TABLE 1: Descriptive characteristics of the sample at baseline and follow-up. Values are mean \pm SD (exept for prevalences).

\begin{tabular}{lcccc}
\hline \multirow{2}{*}{$\begin{array}{l}\text { f(male, } \\
\text { female) }\end{array}$} & $\begin{array}{c}\text { Intervention } \\
(244,201)\end{array}$ & $\begin{array}{c}\text { Control } \\
(259,231)\end{array}$ & $\begin{array}{c}\text { Intervention } \\
(244,201)\end{array}$ & $\begin{array}{c}\text { Control } \\
(259,231)\end{array}$ \\
\hline Age (years) & $7.6 \pm 0.4$ & $7.5 \pm 0.4$ & $8.8 \pm 0.4$ & $8.8 \pm 0.4$ \\
Height $(\mathrm{cm})$ & $127.4 \pm 5.9$ & $127.0 \pm 5.4$ & $133.9 \pm 6.3$ & $134.1 \pm 5.7$ \\
Weight (kg) & $26.5 \pm 5.1$ & $26.4 \pm 4.8$ & $30.6 \pm 6.5$ & $30.9 \pm 6.0$ \\
BMIPCT & $49.1 \pm 29.4$ & $51.0 \pm 28.1$ & $51.3 \pm 29.9$ & $53.6 \pm 28.8$ \\
$\%$ & $13.9 \%$ & $10.4 \%$ & $14.6 \%$ & $13.9 \%$ \\
$\begin{array}{l}\text { Overweight/ } \\
\text { obese }\end{array}$ & & & & \\
\hline
\end{tabular}

partial $\left.\varepsilon^{2}=0.01\right)$. IAF increased significantly over time, and even though values were higher in the intervention group during baseline and follow-up, the difference became less pronounced at follow-up (Figure 1).

Similar to the repeated measures ANCOVA, no difference in BMIPCT was observed at follow-up when controlling for sex, age, and baseline BMIPCT. The control group, however, displayed a significantly higher total CVD risk score, after controlling for sex, age and baseline CVD risk $(F(1,643)=$ 5.14, $P=0.02$, partial $\left.\varepsilon^{2}=0.01\right)$. This result, however, was no longer significant after additionally controlling for BMIPCT. Regarding individual CVD risk factors, only MAP was significantly higher in the control group compared to the intervention group when controlling for sex, age, and baseline values $\left(F(1,902)=11.36, P<0.01\right.$, partial $\left.\varepsilon^{2}=0.01\right)$, and this result remained after additionally controlling for BMIPCT. Analysing changes in the control and intervention groups separately over time, an increase in MAP was shown in the control group $(t(463)=-5.87, P<0.01)$ while MAP remained stable in the intervention group. IAF and BMIPCT increased in both groups $(P<0.01)$ while no significant change in TC: HDL ratio occurred in either group. Overall CVD risk, however, decreased significantly in the intervention group $(t(306)=2.31, P=0.02)$, while it increased in the control group $(t(335)=-1.97, P=0.05)$.

Regarding health behaviour, a significant reduction in the consumption of sugar-sweetened beverages from baseline to follow-up was observed in the intervention group $(t(324)=$ 5.55, $P<0.01$ ), while no change occurred in the control group. Sports participation declined in the control group $(t(388)=2.65, P<0.01)$, while it remained stable in the intervention group. Time spent playing outside, on the other hand, was reduced in the control as well as the intervention groups $(P<0.01)$. Nevertheless, children in the intervention group displayed a higher odds ratio for playing outside at follow-up when controlling for sex, age, and baseline values (Table 2). They also had reduced odds for regular consumption of sugar-sweetened beverages. For sedentary behaviour, an increase in TV time occurred in the control group $(t(442)=-2.04, P=0.04)$ but not in the intervention group. Time spent on the computer increased in both groups $(P<0.01)$. Odds ratios for sedentary behavior were not significant, but a trend towards a lower odds ratio for high 


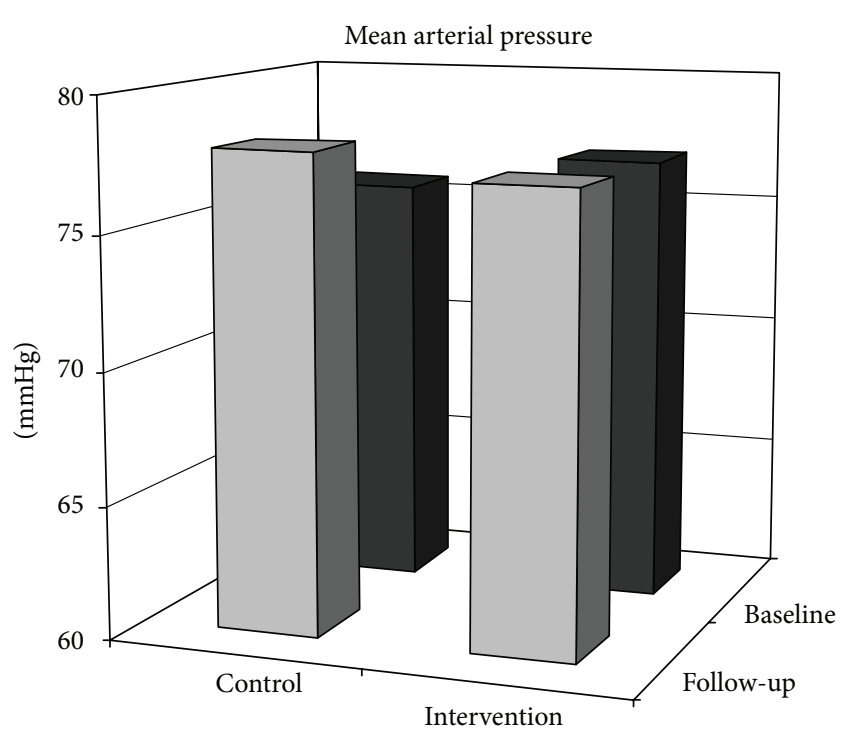

(a)

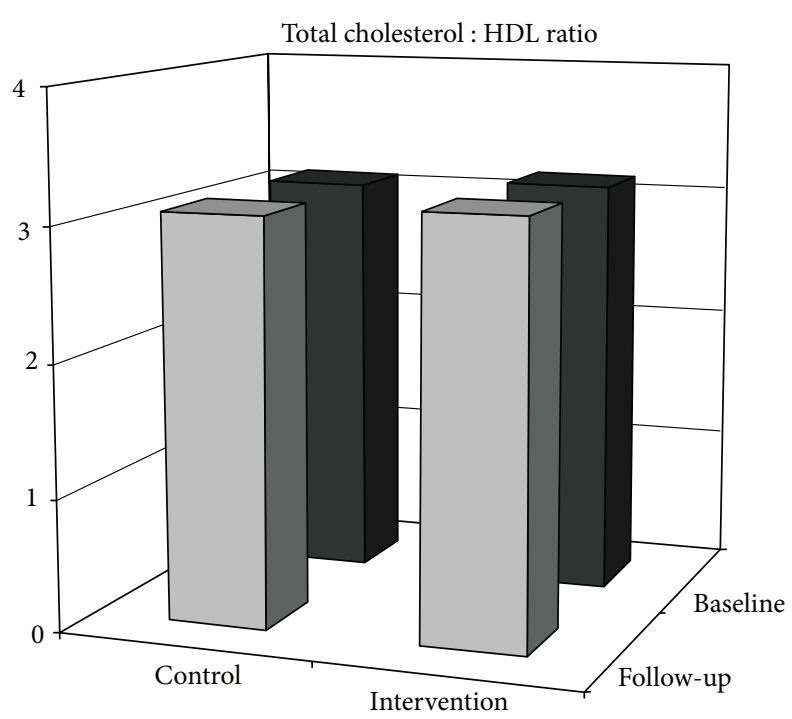

(c)

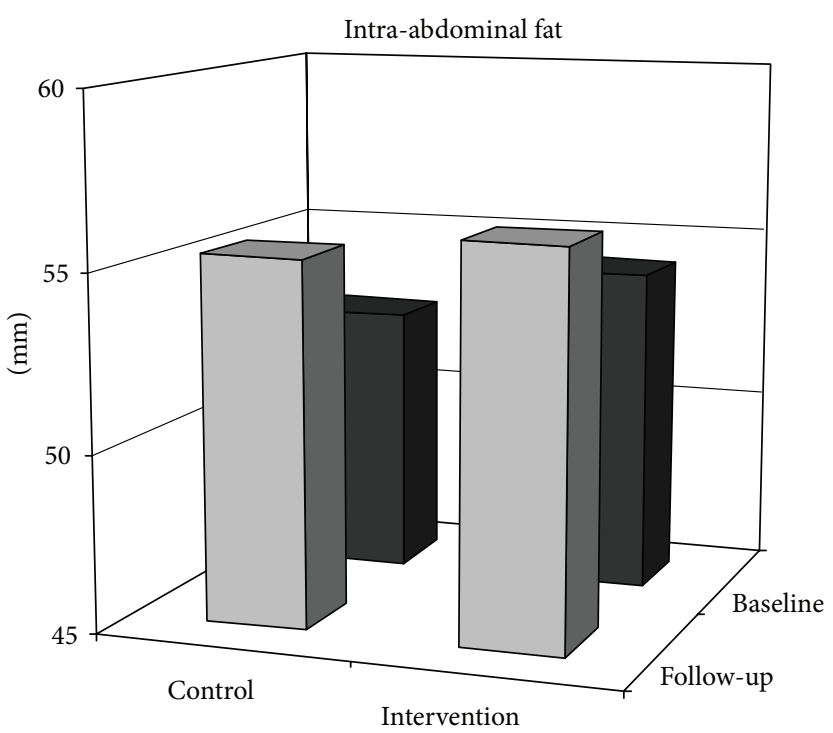

(b)

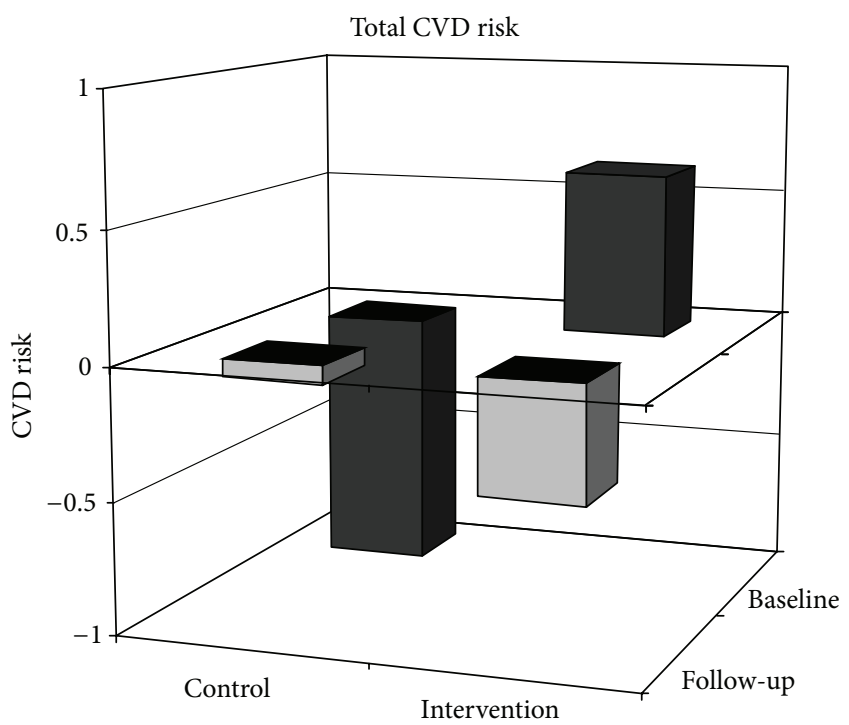

(d)

FIGURE 1: Cardiovascular risk factors and total cardiovascular risk in the control and intervention groups during baseline and follow-up assessments. Values are means adjusted for sex, age, and BMIPCT.

sedentary behaviour was observed in the intervention group (Table 2).

\section{Discussion}

Even though effects were relatively small, the results of this study indicate that a teacher-implemented intervention, which emphasizes parental involvement, has the potential to affect health behavior and reduce CVD risk. Despite a lack of effect on body composition, the intervention reduced CVD risk in these elementary school children. Specifically, an attenuation in the age-related increase in MAP was observed. There was also an attenuation of the age related decline in habitual sports participation in the intervention group.
Further, a decline in the consumption of sugar-sweetened beverages was observed in the intervention group resulting in reduced odds for regular consumption of sugar-sweetened beverages following intervention. The results also indicate a positive effect on sedentary behavior as TV time only increased in the control group. Even though both groups increased their time spent on the computer, the increase was smaller in the intervention group.

Sahota et al. did not report an effect of school-based health promotion programs on body composition either, which was explained by the duration of the intervention [23]. These authors argue that, despite a successful implementation in schools, an intervention period of one year would not be long enough to expect any significant changes in BMI. Other studies, however, reported significant effects with obesity 
TABLE 2: Odds ratio, adjusted for sex, age, and baseline value, of health behaviors in the intervention group compared to the control group at follow-up. Significant results are in bold.

\begin{tabular}{lcc}
\hline & Odds ratio & $\begin{array}{c}95 \% \text { confidence } \\
\text { interval }\end{array}$ \\
\hline $\begin{array}{l}\text { Consumption of } \\
\text { sugar-sweetened beverages } \\
\text { (regular) }\end{array}$ & $\mathbf{0 . 6 5}$ & $\mathbf{0 . 4 6 ; 0 . 9 2}$ \\
$\quad \begin{array}{l}\text { Regular breakfast } \\
\text { Playing outside (almost daily) }\end{array}$ & 1.17 & $0.71 ; 1.93$ \\
$\quad$ Sports participation & $\mathbf{1 . 4 1}$ & $\mathbf{1 . 0 2 ; 1 . 9 4}$ \\
$\quad$ (at least once a week) & 1.06 & $0.76 ; 1.46$ \\
$\quad$ TV time (>60 min/day) & 0.81 & $0.59 ; 1.13$ \\
$\quad$ Computer time $(>30$ min/day) & 0.88 & $0.58 ; 1.11$ \\
\hline
\end{tabular}

prevention programs in schools, particularly when parents were included [24-26]. Nevertheless, Shaya et al. questioned the sustainability of these short-term effects in response to school-based interventions over a prolonged period of time [25], and a Cochrane review reported little evidence for the effect of school-based interventions on body weight beyond the intervention period [27]. The necessity of a prolonged engagement in an intervention to observe changes in body weight was also indicated by Taylor et al. who reported lower BMI values in children who participated in a schoolbased intervention for at least 1 year [28]. For a significant reduction in the prevalence of obesity, however, 2 years of the intervention were necessary. As the intervention program in the present study was implemented by the classroom teacher, a prolonged engagement with the intervention should be possible, and Waters et al. concluded that, especially at the ages of 6 through 12, such interventions would be beneficial despite the relatively small effects observed initially [29]. Similarly, Brown and Summerbell emphasized a combination of dietary information and physical activity in school-based interventions to successfully prevent the development of overweight or obesity in the longterm even though changes may not be visible within one year of the intervention [12].

It should also be considered that body weight is not the only CVD risk factor. At this time, however, only limited research on the effect of school-based interventions on other markers of CVD risk is available. Naylor and McKay showed a reduction in CVD risk and an increase in bone health with the implementation of active breaks during regular class time [6], and other studies showed that school-based interventions can attenuate the age-related increase in MAP in children and adolescents [30-32], which is consistent with the findings of the present study. Results on the effect of school-based interventions on cholesterol levels are less consistent. The present study as well as Harrell et al. did not show any changes in blood lipids within one year [32], while Manios et al. did report a significant difference in cholesterol levels [33]. In this study, however, participants engaged in the intervention for over 6 years, which suggests that longer intervention periods might be necessary to achieve changes in blood lipids. Similarly, results on changes in body fat in response to school-based intervention programs have been inconsistent. Several studies have shown an attenuation in skinfold thickness with a school-based intervention [30, 33], and Rush et al. reported a reduction in the accumulation of body fat, especially in younger children [31]. Harris et al., on the other hand, concluded in their literature review, that activity-based interventions are ineffective in improving body composition or body fatness [15], and Bronikowski and Bronikowska did not show any differences in body fatness 15 months after the cessation of an intervention in adolescents [34]. These authors, however, showed that physical activity levels remained higher in the intervention group, which was also shown in the younger sample of the present study.

Verstraete et al. also showed an attenuation of the agerelated decline in moderate PA and moderate-to-vigorous PA via accelerometry [35]. Further, the incorporation of at least one activity break per day during class time was shown to increase daily step counts in elementary school children [36]. Higher PA levels, however, could not always be sustained beyond the intervention [37], and to ensure at least a modest increase in PA levels, environmental changes in addition to an education-only approach are suggested [6]. Increased awareness on the importance of a healthy lifestyle could further affect sedentary behavior and dietary patterns [26]. Results of the present study support this argument as children in the intervention group were able to maintain their habitual sports participation and TV time, while children in the control group increased their TV time and reduced their PA. In addition, a reduction in the consumption of sugarsweetened beverages was reported, which has been shown previously as well even if no change in the intake of fruits and vegetables was observed [38]. Including the community as well as the family in a school-based intervention did result in an increase in fruit and vegetable consumption in addition to a decline in screentime [39]. Providing information on the caloric content of sugar-sweetened beverages in stores rather than education in schools was also successful in reducing the consumption of sugar-sweetened beverages in adolescents [40].

The lack of consistent results on effects of school-based interventions to promote health behavior may partially be due to different intervention protocols but could also be a result of differences in the assessment of the variables of interest. Due to the young age of the participants, the present study relied on parent report, which could lead to biased results. As parents knew about the goal of the intervention, they might have responded in a more socially desirable way. It is, however, unlikely that only parents of the intervention group displayed such a desirability bias [39]. Even though only limited information on actual parental involvement in the present study is available, parental involvement could be an important aspect in the establishment of a healthy lifestyle in children. Participation in the family homework assignments and knowledge gained via parent-teacher meetings may even provide some benefits for the parents themselves.

In contrast to the subjective report on health behavior, indicators for CVD risk were assessed objectively, and results indicate the potential for a reduction of CVD risk with a teacher-centred intervention. After one year of the intervention, effects were only shown for MAP, but a prolonged engagement in the intervention may result in further positive 
effects on markers of CVD risk. Since the intervention was incorporated in the regular curriculum and delivered by the classroom teacher, it should be sustainable over a prolonged period of time [41]. While higher-dose interventions potentially yield more pronounced results in the short term, they may not allow for long-term sustainability. Long-term interventions involving the parents in addition to educational and environmental adjustments in the school, however, are suggested to ensure a healthier overall lifestyle that would track into adolescence and possibly even adulthood [33]. It should also be mentioned that the reduction in educational minutes due to active breaks does not affect academic performance in children [42]. Activity breaks in the educational setting have actually been shown to increase academic performance [43] and positively affect children's attention [44], which should increase the adherence of teachers who are implementing the intervention program.

\section{Conclusions}

In conclusion, results of the present study show a modest effect on CVD risk and health behavior in elementary school children. As the intervention was incorporated by the classroom teacher within the regular curriculum, a prolonged engagement beyond the evaluation period should be possible, and the sustainability of this program throughout the entire elementary school period could have more pronounced effects in the long term, possibly influencing BMI and overweight or obesity rates [39]. In an international review Dobbins et al. concluded that school-based interventions positively affect sedentary behavior, aerobic fitness, and blood cholesterol, despite a lack of evidence for positive effects on body composition [45]. Even though results of the present study are promising, it should be considered that no single intervention fits all schools, and programs should be adjusted according to the target population [24]. Girls, for example, are more responsive to educational interventions, while boys show a better response to structural and environmental changes that facilitate increased physical activity or a healthier diet [14]. Not surprisingly, participants who are more engaged in the intervention have been shown to display better results [39]. Intervention strategies, therefore, need to consider the needs and requirements of the target population to be successful. Independent of the chosen intervention strategy, the sustainability of the intervention program over a prolonged period of time is a crucial aspect to ensure clinically relevant changes that can be maintained beyond the intervention period. A teacher-implemented health promotion program is one approach to address the growing problems associated with a sedentary lifestyle and poor diet choices in elementary school children.

\section{Acknowledgments}

The study has been funded by the Baden-Wurttemberg Stiftung, Stuttgart, Germany. The URMEL-ICE-study group was headed by Jürgen M. Steinacker, Division of Sportsand Rehabilitation Medicine; Martin Wabitsch and Christoph
Galm, Department of Pediatrics; Richard Peter and Jochen Klenk, Institute of Epidemiology; Michael Fritz, Transfer Center for Neurosciences and Learning; all University of Ulm, Germany. The authors would like to acknowledge and thank all members of the study team for their excellent work and the teachers and pupils who participated in the study.

\section{References}

[1] I. Pigeot, C. Buck, D. Herrmann, and W. Ahrens, "Overweight and obesity in children and adolescents. The worldwide situation," Bundesgesundheitsblatt Gesundheitsforschung Gesundheitsschutz, vol. 53, no. 7, pp. 653-665, 2010.

[2] World Health Organisation, "World health report 2002: reducing risk-promoting healthy life," Tech. Rep., WHO Press, Geneva, Switzerland, 2002.

[3] W. H. Dietz, "Health consequences of obesity in youth: childhood predictors of adult disease," Pediatrics, vol. 101, no. 3, pp. 518-525, 1998.

[4] A. S. Singh, C. Mulder, J. W. R. Twisk, W. Van Mechelen, and M. J. M. Chinapaw, "Tracking of childhood overweight into adulthood: a systematic review of the literature," Obesity Reviews, vol. 9, no. 5, pp. 474-488, 2008.

[5] D. J. Gunnell, S. J. Frankel, K. Nanchahal, T. J. Peters, and G. D. Smith, "Childhood obesity and adult cardiovascular mortality: a 57-y follow-up study based on the Boyd Orr cohort," American Journal of Clinical Nutrition, vol. 67, no. 6, pp. 1111-1118, 1998.

[6] P.-J. Naylor and H. A. McKay, "Prevention in the first place: schools a setting for action on physical inactivity," British Journal of Sports Medicine, vol. 43, no. 1, pp. 10-13, 2009.

[7] L. J. Ignarro, M. L. Balestrieri, and C. Napoli, "Nutrition, physical activity, and cardiovascular disease: an update," Cardiovascular Research, vol. 73, no. 2, pp. 326-340, 2007.

[8] C. D. Summerbell, "The identification of effective programs to prevent and treat overweight preschool children," Obesity, vol. 15, no. 6, pp. 1341-1342, 2007.

[9] R. E. Rhodes, H. M. Macdonald, and H. A. McKay, "Predicting physical activity intention and behaviour among children in a longitudinal sample," Social Science and Medicine, vol. 62, no. 12, pp. 3146-3156, 2006.

[10] R. E. Rhodes, R. C. Plotnikoff, and K. S. Courneya, "Predicting the physical activity intention-behavior profiles of adopters and maintainers using three social cognition models," Annals of Behavioral Medicine, vol. 36, no. 3, pp. 244-252, 2008.

[11] I. De Bourdeaudhuij, E. Van Cauwenberghe, H. Spittaels et al., "School-based interventions promoting both physical activity and healthy eating in Europe: a systematic review within the HOPE project," Obesity Reviews, vol. 12, no. 3, pp. 205-216, 2011.

[12] T. Brown and C. Summerbell, "Systematic review of schoolbased interventions that focus on changing dietary intake and physical activity levels to prevent childhood obesity: an update to the obesity guidance produced by the National Institute for Health and Clinical Excellence," Obesity Reviews, vol. 10, no. 1, pp. 110-141, 2009.

[13] W. Zenzen and S. Kridli, "Integrative review of school-based childhood obesity prevention programs," Journal of Pediatric Health Care, vol. 23, no. 4, pp. 242-258, 2009.

[14] J. A. Kropski, P. H. Keckley, and G. L. Jensen, "Schoolbased obesity prevention programs: an evidence-based review," Obesity, vol. 16, no. 5, pp. 1009-1018, 2008. 
[15] K. C. Harris, L. K. Kuramoto, M. Schulzer, and J. E. Retallack, "Effect of school-based physical activity interventions on body mass index in children: a meta-analysis," Canadian Medical Association Journal, vol. 180, no. 7, pp. 719-726, 2009.

[16] J. J. Reilly, E. Methven, Z. C. McDowell et al., "Health consequences of obesity," Archives of Disease in Childhood, vol. 88, no. 9, pp. 748-752, 2003.

[17] J.-P. Després, I. Lemieux, J. Bergeron et al., "Abdominal obesity and the metabolic syndrome: contribution to global cardiometabolic risk," Arteriosclerosis, Thrombosis, and Vascular Biology, vol. 28, no. 6, pp. 1039-1049, 2008.

[18] L. K. Bartholomew, G. Parcel, G. Kok, and N. Gottlieb, Intervention Mapping: Developing Theory and Evidence-Based Health Education Programs, Mayfield, Mountain View, Calif, USA, 2001.

[19] K. Kromeyer-Hauschild, M. Wabitsch, D. Kunze et al., "Perzentile für den Body-mass-Index für das Kindes- und Jugendalter unter Heranziehung verschiedener deutscher Stichproben," Monatsschrift Kinderheilkunde, vol. 149, pp. 807-818, 2001.

[20] National High Blood Pressure Education Program Working Group on High Blood Pressure in Children and Adolescents, "The fourth report on the diagnosis, evaluation, and treatment of high blood pressure in children and adolescents," Pediatrics, vol. 114, no. no. 2, supplement, 4th report, pp. 555-576, 2004.

[21] F. Armellini, M. Zamboni, L. Rigo et al., "The contribution of sonography to the measurement of intra-abdominal fat," Journal of Clinical Ultrasound, vol. 18, no. 7, pp. 563-567, 1990.

[22] B.-M. Kurth, "The German Health Interview and Examination Survey for Children and Adolescents (KiGGS): an overview of its planning, implementation and results taking into account aspects of quality management," Bundesgesundheitsblatt Gesundheitsforschung Gesundheitsschutz, vol. 50, no. 5-6, pp. 533-546, 2007.

[23] P. Sahota, M. C. J. Rudolf, R. Dixey, A. J. Hill, J. H. Barth, and J. Cade, "Randomised controlled trial of primary school based intervention to reduce risk factors for obesity," British Medical Journal, vol. 323, no. 7320, pp. 1029-1032, 2001.

[24] A. Z. Khambalia, S. Dickinson, L. L. Hardy, T. Gill, and L. A. Baur, "A synthesis of existing systematic reviews and meta-analyses of school-based behavioural interventions for controlling and preventing obesity," Obesity Reviews, vol. 13, no. 3, pp. 214-233, 2012.

[25] F. T. Shaya, D. Flores, C. M. Gbarayor, and J. Wang, "Schoolbased obesity interventions: a literature review," Journal of School Health, vol. 78, no. 4, pp. 189-196, 2008.

[26] S. L. Gortmaker, K. Peterson, J. Wiecha et al., "Reducing obesity via a school-based interdisciplinary intervention among youth: planet Health," Archives of Pediatrics and Adolescent Medicine, vol. 153, no. 4, pp. 409-418, 1999.

[27] C. D. Summerbell, E. Waters, L. D. Edmunds, S. Kelly, T. Brown, and K. J. Campbell, "Interventions for preventing obesity in children," Cochrane Database of Systematic Reviews, no. 3, Article ID CD001871, 2005.

[28] R. W. Taylor, K. A. McAuley, W. Barbezat, V. L. Farmer, S. M. Williams, and J. I. Mann, "Two-year follow-up of an obesity prevention initiative in children: the APPLE project," American Journal of Clinical Nutrition, vol. 88, no. 5, pp. 1371-1377, 2008.

[29] E. Waters, A. de Silva-Sanigorski, B. J. Hall et al., "Interventions for preventing obesity in children," Cochrane Database of Systematic Reviews, vol. 12, Article ID CD001871, 2011.

[30] R. G. Mcmurray, J. S. Harrell, S. I. Bangdiwala, C. B. Bradley, S. Deng, and A. Levine, "A school-based intervention can reduce body fat and blood pressure in young adolescents," Journal of Adolescent Health, vol. 31, no. 2, pp. 125-132, 2002.

[31] E. Rush, P. Reed, S. McLennan, T. Coppinger, D. Simmons, and D. Graham, "A school-based obesity control programme: project Energize. Two-year outcomes," British Journal of Nutrition, vol. 107, no. 4, pp. 581-587, 2012.

[32] J. S. Harrell, S. A. Gansky, R. G. McMurray, S. I. Bangdiwala, A. C. Frauman, and C. B. Bradley, "School-based interventions improve heart health in children with multiple cardiovascular disease risk factors," Pediatrics, vol. 102, no. 2, part 1, pp. 371380, 1998.

[33] Y. Manios, J. Moschandreas, C. Hatzis, and A. Kafatos, "Health and nutrition education in primary schools of Crete: changes in chronic disease risk factors following a 6-year intervention programme," British Journal of Nutrition, vol. 88, no. 3, pp. 315324, 2002.

[34] M. Bronikowski and M. Bronikowska, "Will they stay fit and healthy? A three-year follow-up evaluation of a physical activity and health intervention in Polish youth," Scandinavian journal of public health, vol. 39, no. 7, pp. 704-713, 2011.

[35] S. J. M. Verstraete, G. M. Cardon, D. L. R. De Clercq, and I. M. M. De Bourdeaudhuij, "A comprehensive physical activity promotion programme at elementary school: the effects on physical activity, physical fitness and psychosocial correlates of physical activity," Public Health Nutrition, vol. 10, no. 5, pp. 477484, 2007.

[36] H. E. Erwin, A. Beighle, C. F. Morgan, and M. Noland, "Effect of a low-cost, teacher-directed classroom intervention on elementary students' physical activity," Journal of School Health, vol. 81, no. 8, pp. 455-461, 2011.

[37] K. T. Magnusson, I. Sigurgeirsson, T. Sveinsson, and E. Johannsson, "Assessment of a two-year school-based physical activity intervention among 7-9-year-old children," International Journal of Behavioral Nutrition and Physical Activity, vol. 8, article 138, 2011.

[38] M. Francis, S. S. D. Nichols, and N. Dalrymple, "The effects of a school-based intervention programme on dietary intakes and physical activity among primary-school children in Trinidad and Tobago," Public Health Nutrition, vol. 13, no. 5, pp. 738-747, 2010.

[39] D. A. Gentile, G. Welk, J. C. Eisenmann et al., "Evaluation of a multiple ecological level child obesity prevention program: switch what you do, view, and chew," BMC Medicine, vol. 7, article 49, 2009.

[40] S. N. Bleich, B. J. Herring, D. D. Flagg, and T. L. GaryWebb, "Reduction in purchases of sugar-sweetened beverages among low-income black adolescents after exposure to caloric information," American Journal of Public Health, vol. 102, no. 2, pp. 329-335, 2012.

[41] D. A. Dzewaltowski, P. A. Estabrooks, G. Welk et al., "Healthy youth places: a randomized controlled trial to determine the effectiveness of facilitating adult and youth leaders to promote physical activity and fruit and vegetable consumption in middle schools," Health Education and Behavior, vol. 36, no. 3, pp. 583600, 2009.

[42] Y. Ahamed, H. Macdonald, K. Reed, P.-J. Naylor, T. LiuAmbrose, and H. Mckay, "School-based physical activity does not compromise children's academic performance," Medicine and Science in Sports and Exercise, vol. 39, no. 2, pp. 371-376, 2007. 
[43] J. E. Donnelly and K. Lambourne, "Classroom-based physical activity, cognition, and academic achievement," Preventive Medicine, vol. 52, pp. S36-S42, 2011.

[44] M. T. Mahar, S. K. Murphy, D. A. Rowe, J. Golden, A. T. Shields, and T. D. Raedeke, "Effects of a classroom-based program on physical activity and on-task behavior," Medicine and Science in Sports and Exercise, vol. 38, no. 12, pp. 2086-2094, 2006.

[45] M. Dobbins, K. De Corby, P. Robeson, H. Husson, and D. Tirilis, "School-based physical activity programs for promoting physical activity and fitness in children and adolescents aged 618," Cochrane Database of Systematic Reviews, no. 1, Article ID CD007651, 2009. 


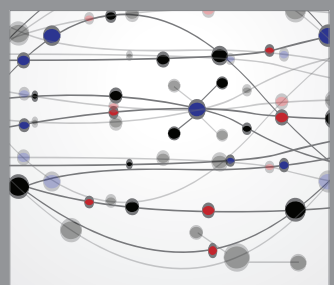

The Scientific World Journal
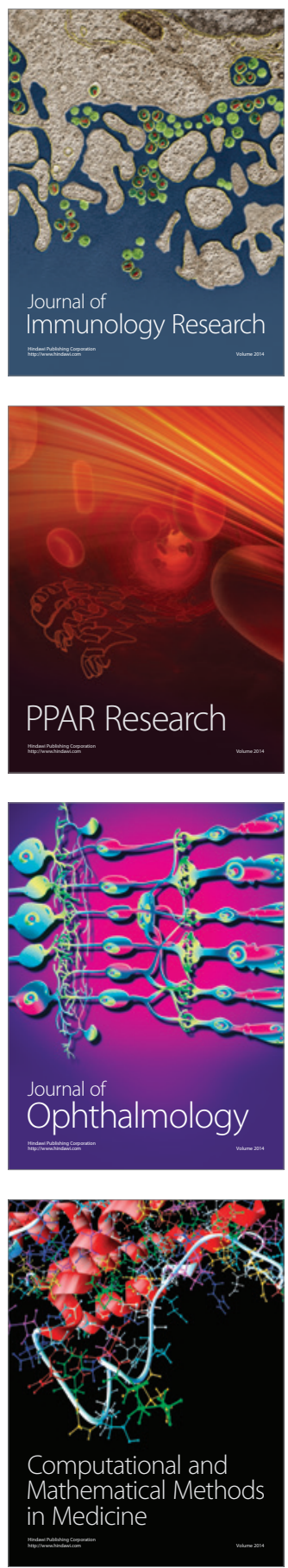

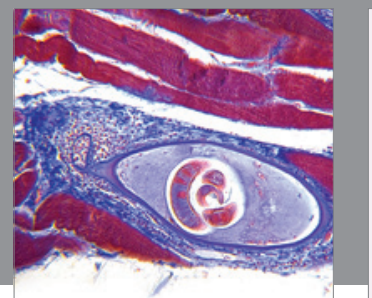

Gastroenterology

Research and Practice
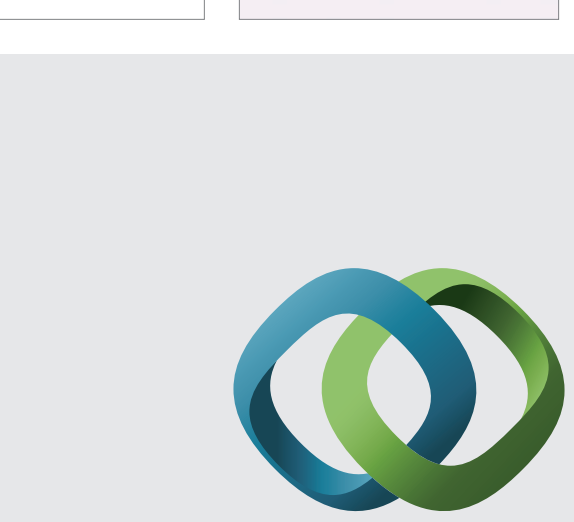

\section{Hindawi}

Submit your manuscripts at

http://www.hindawi.com
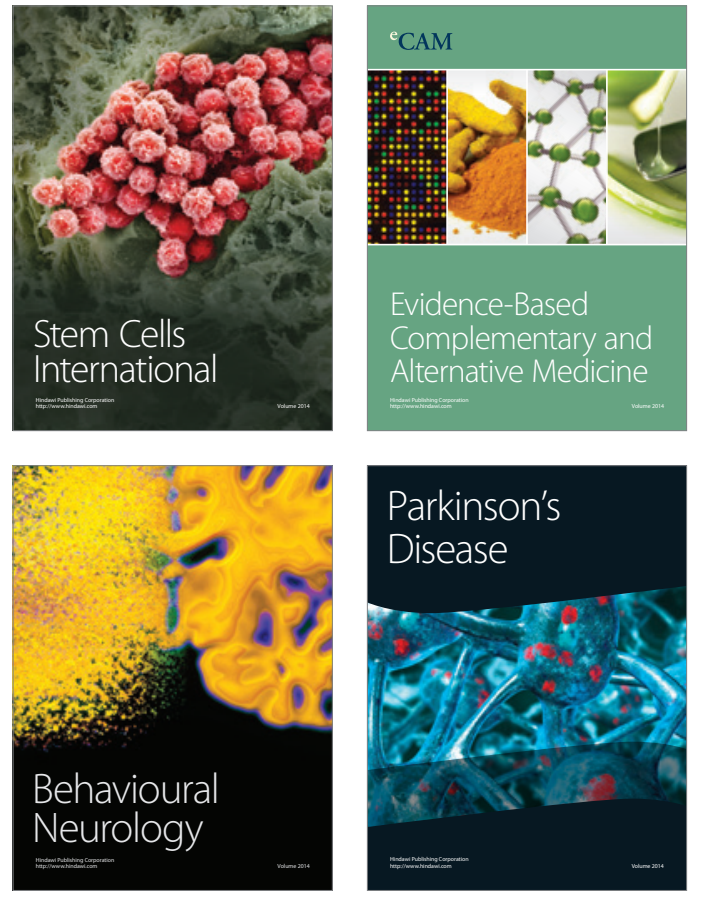
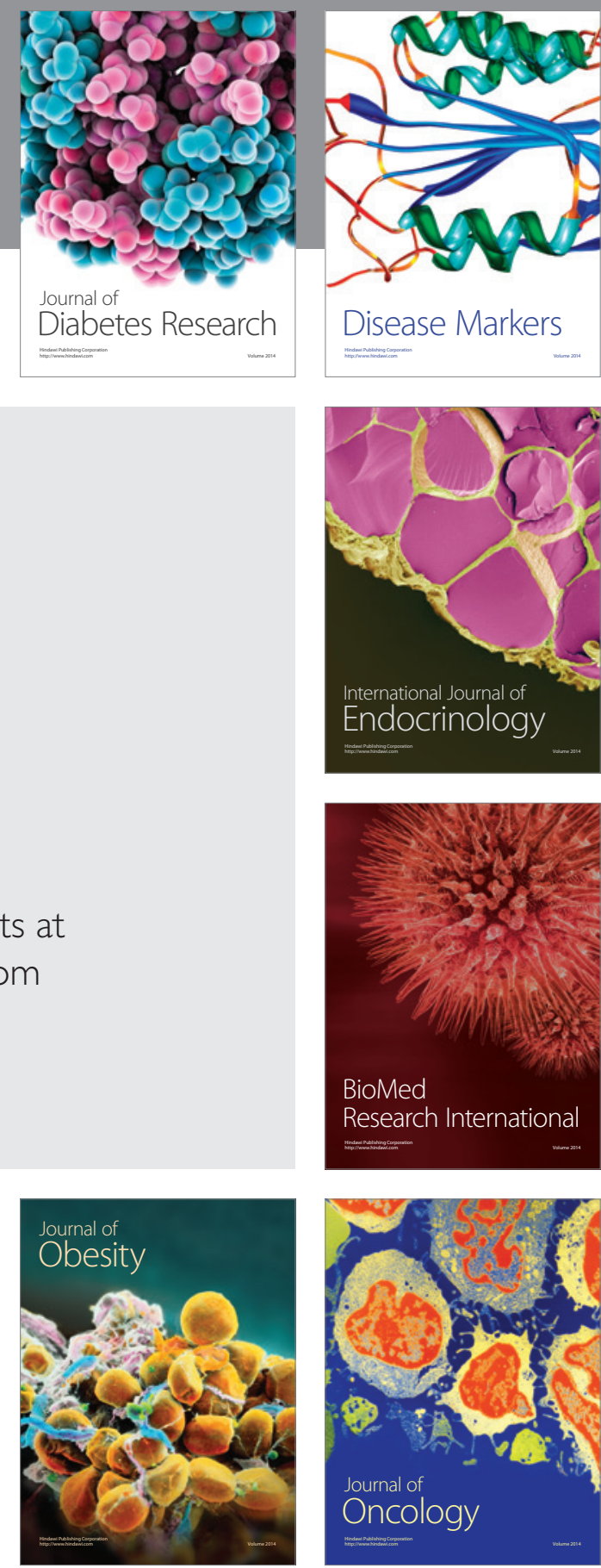

Disease Markers
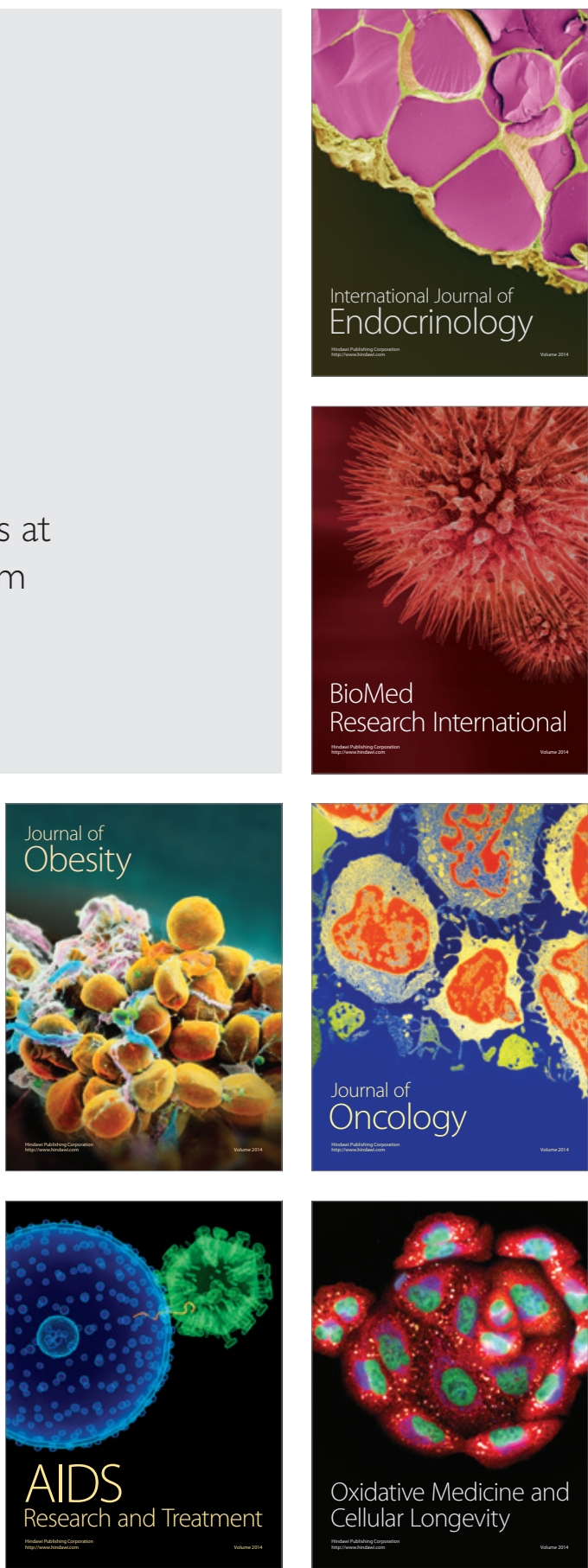